\title{
Impact of a One-Stop Rapid Access Venous Ulcer Clinic on Inpatient Admissions
}

\author{
Colum Keohane ${ }^{1}$, Momhammed Alagha ${ }^{1}$, Marie O’Shaughnessy ${ }^{2}$, Doireann Joyce $^{1}$, Wael \\ Tawfick $^{3}$, Muhammad Tubassam ${ }^{1}$, and STEWART WALSH ${ }^{3}$ \\ ${ }^{1}$ Galway University Hospitals \\ ${ }^{2}$ Roscommon University Hospital \\ ${ }^{3}$ National University of Ireland Galway
}

May 12, 2021

\begin{abstract}
Abstract Objective To determine whether the introduction of a one-stop see and treat clinic offering early reflux ablation for Venous Leg Ulcer (VLU) patients in July 2016 has affected rates of unplanned inpatient admissions due to venous ulceration. Design Review of inpatient admission data and analysis of related costs. Materials The Hospital Inpatient Enquiry collects data from acute public hospitals in Ireland on admissions and discharges, coded by diagnosis and acuity. This was the primary source of all data relating to admissions and length of stay. Costs were calculated from data published by the Health Service Executive in Ireland on average costs per inpatient stay for given diagnosis codes. Methods Data were collected on admission rates, length of stay, overall bed day usage, and costs across a four-year period; the two years since the introduction of the rapid access clinic, and the two years immediately prior as a control. Results 218 patients admitted with VLUs accounted for a total of 2,529 inpatient bed-days, with 4.5(2-6) unplanned admissions, and a median hospital stay of 7(4-13) days per month. Median unplanned admissions per month decreased from $6(2.5-8.5)$ in the control period, to 3.5(2-5) after introduction of the clinic $\mathrm{p}=.040$. Bedday usage was significantly reduced from median $62.5(27-92.5)$, to $36.5(21-44)$ bed-days per month $(\mathrm{p}=.035)$, though length of stay remained unchanged $(\mathrm{p}=.57)$. Cost of unplanned inpatient admissions fell from median $\backslash$ euro33,336.25( $\backslash$ euro14,401.26$\backslash$ euro49,337.65) per month to \euro19,468.37(\euro11,200.98-\euro22,401.96) ( $\mathrm{p}=.03)$. Conclusions Admissions for inpatient management of VLUs have fallen after beginning aggressive endovenous treatment of venous reflux in a dedicated one-stop see-and-treat clinic for these patients. As a result, bed-day usage has also fallen, leading to cost savings.
\end{abstract}

Impact of a One-Stop Rapid Access Venous Ulcer Clinic on Inpatient Admissions

Abstract

Objective

To determine whether the introduction of a one-stop see and treat clinic offering early reflux ablation for Venous Leg Ulcer (VLU) patients in July 2016 has affected rates of unplanned inpatient admissions due to venous ulceration.

\section{Design}

Review of inpatient admission data and analysis of related costs.

\section{Materials}

The Hospital Inpatient Enquiry collects data from acute public hospitals in Ireland on admissions and discharges, coded by diagnosis and acuity. This was the primary source of all data relating to admissions 
and length of stay. Costs were calculated from data published by the Health Service Executive in Ireland on average costs per inpatient stay for given diagnosis codes.

\section{Methods}

Data were collected on admission rates, length of stay, overall bed day usage, and costs across a four-year period; the two years since the introduction of the rapid access clinic, and the two years immediately prior as a control.

\section{Results}

218 patients admitted with VLUs accounted for a total of 2,529 inpatient bed-days, with 4.5(2-6) unplanned admissions, and a median hospital stay of 7(4-13) days per month. Median unplanned admissions per month decreased from 6(2.5-8.5) in the control period, to 3.5(2-5) after introduction of the clinic $\mathrm{p}=.040$. Bedday usage was significantly reduced from median 62.5(27-92.5), to 36.5(21-44) bed-days per month ( $\mathrm{p}=.035)$, though length of stay remained unchanged $(\mathrm{p}=.57)$. Cost of unplanned inpatient admissions fell from median

\section{Conclusions}

Admissions for inpatient management of VLUs have fallen after beginning aggressive endovenous treatment of venous reflux in a dedicated one-stop see-and-treat clinic for these patients. As a result, bed-day usage has also fallen, leading to cost savings.

What is already known about this topic? Early venous reflux ablation can improve the time it takes for venous leg ulcers to heal

What does this article add?

This article shows an association between improved access to specialist services for venous leg ulcer management, including early ablation, and a reduction in inpatient admissions for ulcer related complications

Introduction

Venous ulceration poses a massive economic burden on health services ${ }^{1-4}$. Compression therapy has been shown to aid the healing of venous ulcers ${ }^{5,6}$. It is time consuming for patients and practitioners however and requires significant community support.

Superficial venous reflux (varicose veins) is frequently present in patients with venous leg ulcers ${ }^{7}$, and surgical treatment of varicose veins, in combination with compression, has been shown to improve the rate of ulcer recurrence. However, the ESCHAR trial did not show any benefit in ulcer healing from surgery ${ }^{8,9}$. Therefore, some guidelines only recommend surgery to reduce recurrence ${ }^{10}$ and commonly patients have surgical intervention after their ulcers are healed. Numerous observational studies ${ }^{11-14}$ and the EVRA (Early Venous Reflux Ablation) Trial ${ }^{15}$ have shown that endovenous treatment of varicose veins may improve ulcer healing, and so in recent years guidelines have begun to reflect this. ${ }^{16,17}$

In specialist practice it is uncommon for patients to be admitted for management of venous ulcers, and it is typically dealt with in an outpatient setting unless there is superimposed infection. It is not unusual however for elderly, comorbid patients to be referred to emergency departments with VLUs, even when infection is not necessarily the issue, and often leads to admission in non-specialist centres. In this population, this can lead to prolonged inpatient stays for patients who could otherwise be managed effectively at home. An alternative pathway, avoiding emergency department referrals and providing rapid access to specialist care might reduce these admissions.

We introduced a 'one-stop see and treat' clinic for venous leg ulcer (VLU) patients in 2016. We aimed to determine how this service impacted upon unplanned hospital admissions for venous ulceration.

Aims 
Our primary aim was to determine whether unplanned inpatient admissions due to venous ulceration were reduced following the introduction of a one-stop see and treat clinic offering early reflux ablation for VLU patients.

Secondary outcomes included median hospital bed-day usage, length of stay, mean cost of hospital stay per admission, and annual cost.

Materials and Methods

\section{Study Setting}

The Saolta Hospital Group comprises seven hospitals in the West of Ireland, caring for a total population of approximately 800000 people. University Hospital Galway (UHG) is the tertiary referral centre for the group, where vascular surgical services for the entire group are based. Four of the hospitals provide elective and acute general surgical care, while the remaining two provide only elective and day case surgical services.

In July 2016, a one-stop venous ulcer clinic was introduced, operating once weekly for a full day from Roscommon University Hospital. Patients are seen and assessed by a vascular clinical nurse specialist and vascular surgeon, with ultrasound assessment of venous disease and suitability for endovenous intervention. Where necessary, further imaging or investigation can be arranged but for the majority, the aim is same day treatment of superficial venous reflux, and/or commencement of compression therapy as appropriate. Suitable patients are offered axial ablation (mechanochemical ablation using Clarivein ${ }^{\mathrm{TM}}$ (Vascular Insights LLC, Quincy, MA, USA)), or foam sclerotherapy (using Fibrovein ${ }^{\text {TM }}$ (STD Pharmaceutical Products Ltd., Hereford, UK)) into the sub-ulcer plexus of veins, as appropriate. Axial ablation is offered to patients with great or small saphenous vein reflux. Foam is offered along with axial ablation if prominent varicose veins between the ulcer and the ablated segment are an issue, in an effort to treat to the lowest possible point of reflux. Treatment with foam alone is offered for perforator reflux, or reflux without discernible superficial venous disease. All of this is done in consultation with the patient, and some, particularly more elderly patients, elect for foam alone as the, perceived, least invasive procedure, while many elect to continue with compression alone. Compression bandaging is offered to all patients with a VLU and superficial or deep venous reflux with no contraindications.

The clinic runs in parallel with a day case operating list for varicose vein interventions. This allows clinic patients undergoing intervention to be added onto the daycase list and undergo same-day intervention. Another advantage of this is that is allows the clinic to be run with only one additional staff member: the clinical nurse specialist. Theatre nurses, secretaries and other personnel do so in addition to their normal duties, reducing costs.

Patients are referred to the VLU clinic from general practice, community wound care services, and from general surgical teams in any of the hospitals within the Saolta group where such services are provided. Patients with venous reflux of any type, without ulceration, were not diverted to the rapid access clinic. Following their initial assessment, and intervention if performed, patients are reviewed every four weeks until their VLU has healed. Where this is not practical for other reasons, most commonly if patients have travelled long distances for assessment and treatment, some patients were followed up locally. If issues arose during follow up, Public Health Nurses (PHNs) were able to contact the clinic nurse directly to arrange rapid reassessment, and indeed patients themselves could make contact directly if necessary.

\section{Data Collection}

The Hospital Inpatient Enquiry (HIPE) collects demographic, clinical and administrative data on discharges from acute public hospitals in Ireland. Data are extracted from medical charts or records and coded according to ICD-10 by trained clinical coders.

Patients were deemed to have an unplanned VLU related admission if they had any non-elective admission, to any hospital within the Saolta group, with an appropriate primary diagnosis code over the period July 
2014-July 2018. The codes for venous ulcer include "Varicose veins of lower extremities with ulcer", and "Varicose veins of lower extremities with both ulcer and inflammation".

Phlebitis alone without ulceration, and varicose veins without ulceration, are both coded elsewhere in the ICD-10 and were excluded. The two years immediately prior to the introduction of the one-stop see and treat clinic (July 2014 to June 2016) served as the control period.

Data on the clinic's activity was gathered by a combination of prospective documentation as well as retrospective chart review for earlier patients.

\section{Statistical Analysis}

Continuous variables were described using means ( \pm standard deviation) for normally distributed data and medians ( \pm interquartile range) for non-parametric data. Categorical variables were described as absolute numbers and percentage frequencies. As length of stay data, and therefore also bed day usage across groups, was non-normally distributed, all analyses relating to these were treated as non-parametric and tested using a Mann-Whitney U. Categorical variables were tested using the Fisher Exact test.

Data was analysed using the StatsDirect ${ }^{\mathrm{TM}}$ software package version 3.

\section{Cost Analysis}

The Irish Health Service Executive (HSE) periodically publishes an inpatient ready reckoner of acute hospital inpatient and daycase activity costs summarised by diagnosis related group (DRG). The most recent edition was published in 2013 using total costs per DRG from 2011 data broken down into costs per average case. Admissions are further stratified by whether a catastrophic or severe complication occurred during their inpatient stay, but as HIPE data does not provide this information, a total average cost per case was calculated. This was then used to calculate cost per bed-day, which was then applied to bed-day usage data for the current study, to calculate monthly costs.

\section{Ethics}

Ethical approval was not sought, in keeping with institutional policy exempting audits and service improvement reviews from ethical approval.

\section{Results}

Over the four-year study period there were 218 VLU-related unplanned, inpatient hospital admissions throughout the Saolta Group. Of these, 122 were coded "Varicose veins of lower extremities with both ulcer and inflammation", while 96 were coded "Varicose veins of lower extremities with ulcer". Overall 131 (59\%) were female and (41\%) male, and the median age was $78.9(69.1-84.9)$. There was no significant difference in likelihood of inflammation being present based on sex $(\mathrm{p}=.09)$ or age $(\mathrm{p}=.15)$.

In total, VLU patients accounted for a total of 2,529 inpatient bed-days, with 4.5(2-6) admissions per month, and a median hospital stay of 7(4-13) days per month throughout this period. Each month, a median 40.5(2171) bed-days were devoted to patients with venous ulceration.

Those with ulcers with inflammation or infection accounted for 1,454days (57.5\%), with a median 8(4-14) days per unplanned admission, while those without accounted for 1,075 days (42.5\%), median 7(3-13) per unplanned admission. There was no significant difference in median length of stay between these groups $(\mathrm{p}=.28)$.

Since commencement of the rapid access clinic, 161 patients were seen with 183 ulcers assessed. 53 of these ulcers were recurrent, and 8 of these recurrences occurred in legs previously treated in the clinic. Demographic data is presented in table 1. In this time, 108/183(59\%) have received endovenous treatment for reflux. Of those, $21(11.5 \%)$ both axial ablation and sclerosant foam were performed for $21(11.5 \%)$ of ulcers, $42(22.9 \%)$ received foam only, and 55(30\%) underwent axial ablation. 46(25.1\%) ulcers were managed with compression alone. The remainder received no treatment of reflux during the study period. 


\section{Admissions}

The primary outcome measure was number of unplanned admissions per month. Over the four-year period and this showed a significant reduction. Median number of unplanned admissions per month decreased from $6(2.5-8.5)$ in the control period, to $3.5(2-5) \mathrm{p}=.04$ after introduction of the clinic. In particular, there was a significant difference between the numbers admitted without inflammation or infection before and after commencement of the service. In the two years prior to the clinic commencing there were 68 admissions without inflammation, and 63 with inflammation. While the numbers admitted with inflammation remained relatively stable at 59, the number admitted without inflammation reduced to $28(\mathrm{p}=.005)$. Point biserial correlation showed a moderate negative correlation between monthly admission rates and whether the month fell before or after the commencement of the clinic $\mathrm{r}=-.32931(\mathrm{p}=.02228)$ In the period since the commencement of the clinic there is a moderate negative correlation between monthly admissions and time since commencement $\mathrm{R}=-0.35$ (two sided $\mathrm{p}=0.0392$ )

\section{Secondary Endpoints}

Length of inpatient stay did not change significantly during the study period $(\mathrm{p}=.57)$, regardless of sex $(\mathrm{p}=.5141)$, or age $(\mathrm{p}=.85)$.

Bed-day usage was significantly reduced from median $62.5(27-92.5)$, to $36.5(21-44)$ bed-days per month $(\mathrm{p}=.035)$.

In 2011, 291 unplanned admissions due to VLU cost the state inpatient stay of \euro7,608, or \euro533.38 per bed-day. The results of applying these costs to the 2014-2018 Saolta data are shown in table 3 and figure 1. Figure 1 shows the median monthly cost borne by the health service for the inpatient care of patients admitted primarily for management of VLUs in the two-year period immediately before and the two-year period since commencement of the VLU clinic.

The cost of management of varicose veins on a surgical daycase basis amounts to \euro2,211 per case. The current data show a median cost per inpatient stay throughout the study period of $\backslash$ euro3,733.66, which did not change significantly, as length of stay was not significantly reduced. There was however a significant reduction in costs per month from median \euro33 336.25( \euro14,401.26-\euro49,337.65) to reduced admission rate.

\section{Discussion}

To our knowledge this study represents the first time that a dedicated see-and-treat service for VLUs has been assessed in terms of its potential to reduce the burden on inpatient services, and therefore costs.

We have shown that ulcer admission rates have fallen after beginning a rapid access clinic providing aggressive treatment of VLUs with surgical intervention. It remains unclear whether rapid access to the actual interventions is wholly responsible for the reduced admissions and observed healing rates do not support this, though it is likely one important factor. We believe that the reduction of inpatient admissions is a result of a combination of factors. These include reduced time from referral to specialist assessment and treatment, greater availability of an alternative referral pathway for primary care physicians, and the safety net effect of frequent follow up and easy access in the event of a setback. The observed saving in bed-days alone suggests there is value providing such a service for management of VLUs, even before financial costs are considered. Cost per admission remained static during the study period, as length of stay per case did not significantly change. The number of admissions however was reduced, leading to significant cost savings as well as freeing up beds. The cost of surgical daycase interventions for varicose veins to the HSE amounts to \euro2,211 per case. While the addition of 108 of these cases into the system since the commencement of the clinic would offset savings made in admission costs, since current guidelines already advocate surgery in these patients for prevention of recurrence, the majority undergo a daycase treatment of their varicose veins

An analysis of the breakdown of costs in VLU management, albeit performed in the US where the funding model is markedly different to the Irish or most European systems, found that just $22 \%$ of the costs relating 
to venous leg ulcer management were incurred in the inpatient setting. The rest of the costs incurred were split between outpatient management $(42 \%)$ and community care $(35 \%) \operatorname{costs}^{18}$.

In these areas the one-stop clinic can also have secondary benefits, and from its inception one of the main goals of the clinic was to streamline the process by which patients with VLUs are managed. A one-stop clinic reduces the number of hospital visits and removes the need for separate waiting lists for assessment and intervention. Reducing the number of visits required (from one for assessment, one for the procedure at a later date, and perhaps another for ultrasound assessment in between) into a single visit reduces the total number of outpatient visits.

Prompt treatment, while not removing the need for community follow up, should also reduce the number of healthcare interactions required by these patients in the community. Improving the ulcer healing time can eliminate at least one public health nurse visit for every extra ulcer-free week, and in some cases 2-3 visits. Accepting the evidence of the EVRA trial, treatment of reflux was offered to all patients in whom it was felt to be appropriate. We do not dispute these data, but perhaps in a real world rather than a trial setting our improvements in healing are not as promising. In addition, as the same-day service removes the waiting period in a typical system between assessment and treatment, public health nurse visits to patients on a surgical waiting list are also saved.

Early and easy access for these patients, also streamlines wound care in the community. More and more of these interactions amount to a simple dressing change, as a full assessment has been carried out already. If an ulcer is thought to be making poor progress, the public health nurses know the patient will be seen within a month and in the event of a problem, they have a definite referral pathway, to have patients about whom there is a concern seen at the next weekly clinic. This removes the dilemma, over whether to send a non-acute patient to the emergency department if the patient cannot wait on a normal outpatient waiting list while an ulcer continues to deteriorate. This is reflected in the evidence presented. While admission rates with inflamed or infected ulcers remained relatively unchanged, there was a marked reduction in admissions of ulcers without inflammation. These patients tend to be those admitted for inpatient management of difficult ulcers, or ulcers resistant to treatment. While many of those with infection will require admission for antibiotics regardless of treatment strategy, the provision of a different referral pathway for the non-infected but difficult to manage cases allows them to remain in the community, contributing to the reduction in admission rates observed.

There were no other significant changes made in service provision in the geographical area in question which account for the significant reduction in inpatient admissions over the course of the study period. We therefore suggest that in light of clinical guidance recommending the surgical management of venous reflux to encourage ulcer healing ${ }^{16,17}$ that this be undertaken in a one-stop clinic to maximise efficiency.

\section{Limitations}

The lack of complete prospective data for all patients seen in the clinic, in particular incomplete data in relation to healing rates within the cohort, limits understanding of the precise mechanism by which the reduction in admissions has occurred.

The data source for this study, HIPE, is collected prospectively, by chart review, by trained clinical coders at the time of discharge. The coders review patient notes, radiology and lab data, as well as discharge summaries, to code each admission. The coders receive clinical training, but the focus of the coding is intended towards finance rather than research. There is the potential for some patients to be incorrectly coded, so HIPE data can be limited in its accuracy for each individual case. However, over the course of hundreds of admissions, as in the NSQIP (National Surgical Quality Improvement Program) database run by the American College of Surgeons or HES (Hospital Episode Statistics) database, in the NHS, these inaccuracies are expected to be evenly distributed.

Perforators were treated with foam sclerotherapy and were not singled out as a separate modality in data collection. 
Conflict of Interest

The authors declare no conflict of interest.

Funding

This research did not receive any specific funding from agencies in the public, commercial, or not-for-profit sectors.

References

1. O'Brien JF, Grace PA, Perry IJ, et al. Prevalence and aetiology of leg ulcers in Ireland. Irish journal of medical science 2000; 169: 110-112. 2000/09/28.

2. Posnett J, Gottrup F, Lundgren H, et al. The resource impact of wounds on health-care providers in Europe. Journal of wound care2009; 18: 154-161. 2009/04/08. DOI: 10.12968/jowc.2009.18.4.41607.

3. Guest JF, Ayoub N, McIlwraith T, et al. Health economic burden that wounds impose on the National Health Service in the UK. BMJ open2015; 5: e009283. 2015/12/09. DOI: 10.1136/bmjopen-2015-009283.

4. Hareendran A, Doll H, Wild DJ, et al. The venous leg ulcer quality of life (VLU-QoL) questionnaire: development and psychometric validation. Wound repair and regeneration : official publication of the Wound Healing Society [and] the European Tissue Repair Society 2007; 15: 465-473. 2007/07/26. DOI: 10.1111/j.1524475X.2007.00253.x.

5. O'Meara S, Cullum N, Nelson EA, et al. Compression for venous leg ulcers. The Cochrane database of systematic reviews 2012; 11: Cd000265. 2012/11/16. DOI: 10.1002/14651858.CD000265.pub3.

6. Singer AJ, Tassiopoulos A and Kirsner RS. Evaluation and Management of Lower-Extremity Ulcers. The New England journal of medicine2017; 377: 1559-1567. 2017/10/19. DOI: 10.1056/NEJMra1615243.

7. Adam DJ, Naik J, Hartshorne T, et al. The diagnosis and management of 689 chronic leg ulcers in a single-visit assessment clinic.European journal of vascular and endovascular surgery : the official journal of the European Society for Vascular Surgery 2003; 25: 462-468. 2003/04/26. DOI: 10.1053/ejvs.2002.1906.

8. Gohel MS, Barwell JR, Taylor M, et al. Long term results of compression therapy alone versus compression plus surgery in chronic venous ulceration (ESCHAR): randomised controlled trial. BMJ (Clinical research ed) 2007; 335: 83. 2007/06/05. DOI: 10.1136/bmj.39216.542442.BE.

9. Barwell JR, Davies CE, Deacon J, et al. Comparison of surgery and compression with compression alone in chronic venous ulceration (ESCHAR study): randomised controlled trial. Lancet (London, England)2004; 363: 1854-1859. 2004/06/09. DOI: 10.1016/s0140-6736(04)16353-8.

10. Wittens C, Davies AH, Baekgaard N, et al. Editor's Choice - Management of Chronic Venous Disease: Clinical Practice Guidelines of the European Society for Vascular Surgery (ESVS). European journal of vascular and endovascular surgery : the official journal of the European Society for Vascular Surgery 2015; 49: 678-737. 2015/04/30. DOI: 10.1016/j.ejvs.2015.02.007.

11. Alden PB, Lips EM, Zimmerman KP, et al. Chronic venous ulcer: minimally invasive treatment of superficial axial and perforator vein reflux speeds healing and reduces recurrence. Annals of vascular surgery 2013; 27: 75-83. 2012/10/23. DOI: 10.1016/j.avsg.2012.06.002.

12. Marston WA, Crowner J, Kouri A, et al. Incidence of venous leg ulcer healing and recurrence after treatment with endovenous laser ablation. Journal of vascular surgery Venous and lymphatic disorders 2017; 5: 525-532. 2017/06/19. DOI: 10.1016/j.jvsv.2017.02.007.

13. Mauck KF, Asi N, Undavalli C, et al. Systematic review and meta-analysis of surgical interventions versus conservative therapy for venous ulcers. Journal of vascular surgery 2014; 60: 60S-70S.e61-62. 2014/05/20. DOI: $10.1016 /$ j.jvs.2014.04.059. 
14. van Gent WB, Hop WC, van Praag MC, et al. Conservative versus surgical treatment of venous leg ulcers: a prospective, randomized, multicenter trial. Journal of vascular surgery 2006; 44: 563-571. 2006/09/05. DOI: 10.1016/j.jvs.2006.04.053.

15. Gohel MS, Heatley F, Liu X, et al. A Randomized Trial of Early Endovenous Ablation in Venous Ulceration. The New England journal of medicine 2018; 378: 2105-2114. 2018/04/25. DOI: 10.1056/NEJMoa1801214.

16. O'Donnell TF, Jr., Passman MA, Marston WA, et al. Management of venous leg ulcers: clinical practice guidelines of the Society for Vascular Surgery (R) and the American Venous Forum. Journal of vascular surgery 2014; 60: 3s-59s. 2014/06/30. DOI: 10.1016/j.jvs.2014.04.049.

17. Lim CS, Baruah M and Bahia SS. Diagnosis and management of venous leg ulcers. BMJ (Clinical research ed) 2018; 362: k3115. 2018/08/16. DOI: 10.1136/bmj.k3115.

18. Ma H, O'Donnell TF, Jr., Rosen NA, et al. The real cost of treating venous ulcers in a contemporary vascular practice. Journal of vascular surgery Venous and lymphatic disorders 2014; 2: 355-361. 2014/10/01. DOI: $10.1016 / \mathrm{j} . j v s v .2014 .04 .006$.

\section{Hosted file}

image1.emf available at https://authorea.com/users/413398/articles/521729-impact-of-a-onestop-rapid-access-venous-ulcer-clinic-on-inpatient-admissions

Figure 1: Median Monthly Cost ( \euro) of inpatient admissions for VLUs in the two years before and two years after introduction of the rapid access clinic Rapid Access Service. X-axis monthly cost ( \euro).

\begin{tabular}{ll}
\hline Baseline characteristic & $\mathrm{N}$ \\
\hline Female & $88(54.6 \%)$ \\
Median Age-years & $73(63-79)$ \\
Median duration of Ulcer at presentation-months & $9(5-24)$ \\
Concurrent PVD & $20(12.4 \%)$ \\
Diabetes & $17(10.5 \%)$ \\
Current Smoker & $10(6.2 \%$ \\
Previous Venous surgery & $27(16.8 \%)$ \\
Obese & $16(9.9 \%)$ \\
\hline
\end{tabular}

Table 1 Demographic Data

\begin{tabular}{ll}
\hline & $\%$ (no. healed/no. followed) \\
\hline Healed ulcer at 6 months & $50.7 \%(34 / 67)$ \\
Healed in compression alone & $54.5 \%(12 / 22)$ \\
Healed with any intervention & $48.9 \%(22 / 45)$ \\
Healed with axial ablation & $66 \%(12 / 18)$ \\
Healed with foam & $66 \%(9 / 15)$ \\
Healed with ablation and foam & $8.3 \%(1 / 12)$ \\
\hline
\end{tabular}

Table 2 Ulcer healing rates by treatment modality among those

followed for 6 months or until ulcer healed

\begin{tabular}{lll}
\hline Year of study & Annual Cost $\backslash$ euro & Median monthly cost $\backslash$ euro \\
\hline Year 1 & 600052.50 & 47470.82
\end{tabular}




\begin{tabular}{lll}
\hline Year of study & Annual Cost $\backslash$ euro & Median monthly cost $\backslash$ euro \\
\hline Year 2 & 268823.52 & 21601.89 \\
Year 3 & 289825.62 & 21168.65 \\
Year 4 & 187216.38 & 16268.09 \\
\hline
\end{tabular}

Table 3 Annual and Monthly Costs \euro) of inpatient admissions per year of study

\section{Hosted file}

Tables Ulcer Admissions.pdf available at https://authorea.com/users/413398/articles/521729impact-of-a-one-stop-rapid-access-venous-ulcer-clinic-on-inpatient-admissions

\section{Hosted file}

Figures Ulcer admissions.pdf available at https://authorea.com/users/413398/articles/521729impact-of-a-one-stop-rapid-access-venous-ulcer-clinic-on-inpatient-admissions 